\title{
Photonic Displays in Augmented Reality
}

\section{Solomon Budnik \\ budnik1@013.net}

Abstract: Our quantum harmonics electronic system can be based on jump-resonance phenomena of nonlinear feedback control systems of any order. The nonlinearities are those whose outputs are single-valued odd functions of the inputs and are independent of frequencies of the photonic inputs. The general conditions under which jump-resonance occurs will be given and the system with saturation nonlinearity will be analyzed. The essential objective is to define the contours on the complex plane for the constant values of system variables, e.g., input amplitude, amplitude ratio, and phase shift.

\section{ELABORATION}

Common Frequency Hopping Spread Spectrum (FHSS) will be upgraded by us in our quantum harmonics system to randomly propagate atomic particles by photonically switching from one signal carrier (quantum tube, see our quantum harmonics paper) to other quantum channels in thereby achieved dynamic equilibrium beyond chaotic interference.

Fermi-Dirac distribution function (see ref. 2) and (electrovacuum) solutions of the Einstein (Einstein-Maxwell) field equations are applicable.Same concept can be applied in our remote quantum loops space display system (see fig. 1) to constitute space computes and TVs (see fig. 2). We accordingly introduce here the notions of rotating quantum spring (fig. 1) to create a quantum ball, as in a ball lightning:

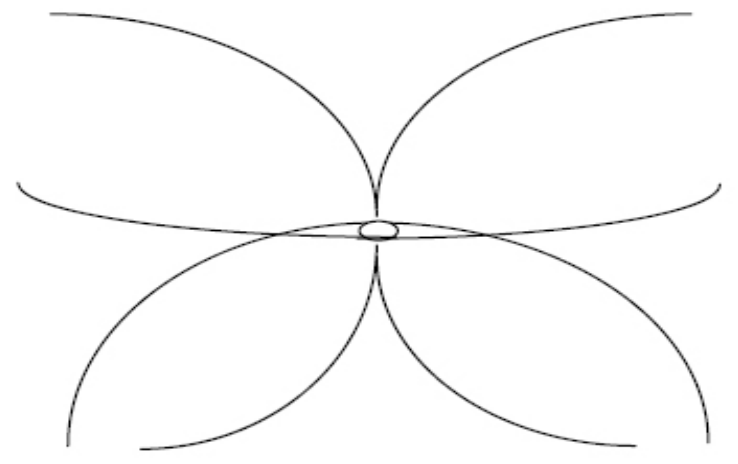

Fig1. Pictured quantum loops with integrated central quantum spring define the contours of their rotating plain as in a ball lightning (ref. 1) to create a 3D space plasma imagery display (fig. 2) in self-generated and contained e.m. field, as in a ball lighting (see ref. 3 Wand 4) to be activated by a tunable pulse laser via quantum prism or membrane.

We show here (fig. 2) the around the axis rotating central quantum spring, which sustains the quantum ball structure:

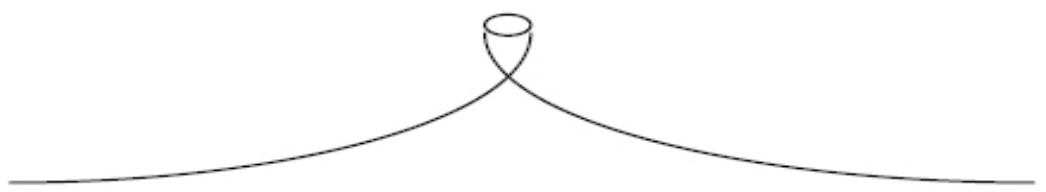

Fig2. Quantum spring 


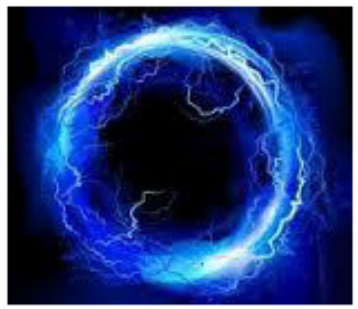

Ill.1. Ball lightning

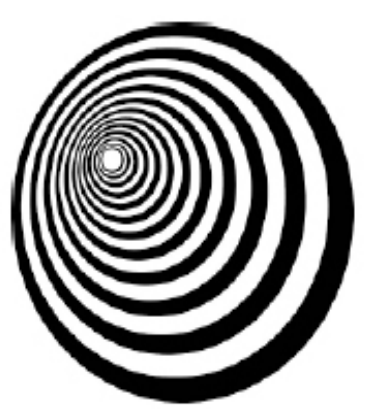

Fig2. 3D quantum space display as in a ball lightning in ref. 1.

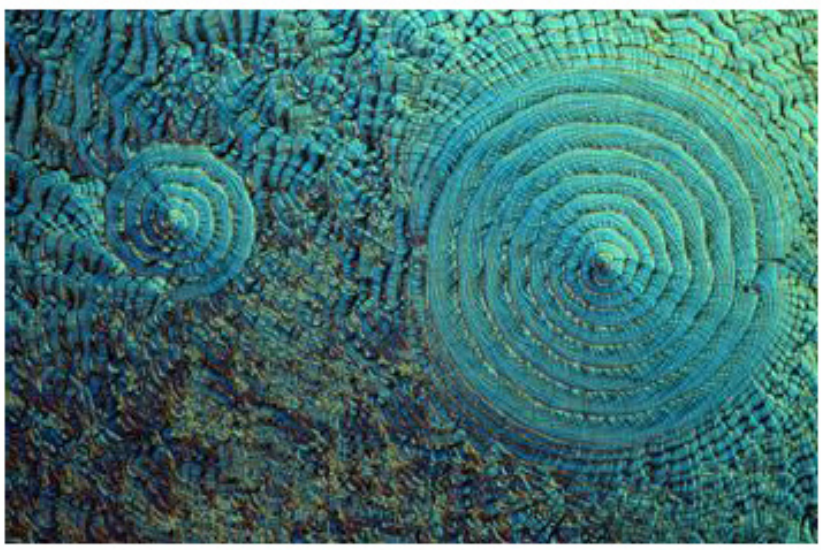

Ill.2. Ball lightning structure

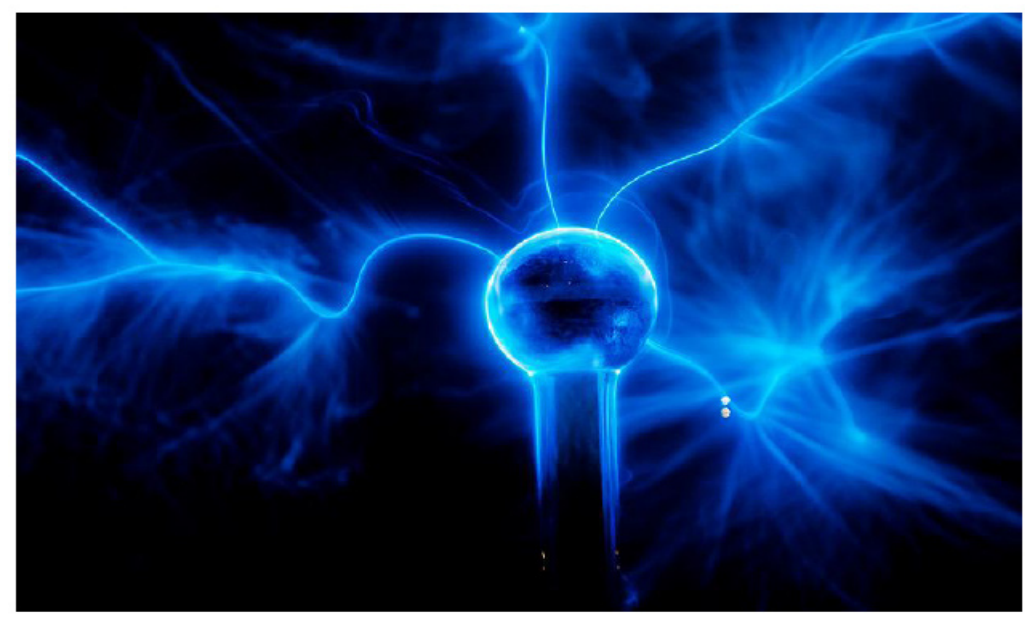

Ill.3. Electron-ionic model of ball lightning 


\section{From Wikiversity}

The electron-ionic model of ball lightning was represented by Sergey G. Fedosin, a physicist and the philosopher from Perm, Russia, and Sergey A. Kim, from Perm state university, in a number of works.

In this model, ball lightning is a cluster of the very hot ionized air with the positive charge in general, whose shell consists of the rapidly revolving electrons with the total current up to $1,4 \bullet 10^{5} \mathrm{~A}$. Ball lightning as whole is supported by the balance of the electromagnetic forces, which act between the charges. Positive ions inside the lightning are distributed freely as a result of the spherical symmetry, and attract to themselves the electrons of shell, retaining them from the dispersion.

According to the model the ball lightning is formed from two close branches of a linear lightning at the time of termination of current in the main channel with the subsequent closure of branches in a current ring.

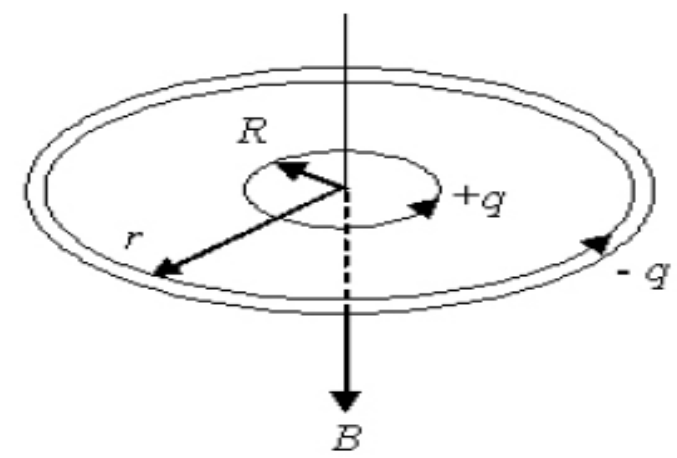

Equatorial cross-section model of ball lightning as a distinct ring on the current sheet spheroidal shape. $\mathrm{R}$ radius of rotation of ions in the equilibrium shell around the magnetic field with induction $B, r$ - radius of the outer electron shell.

Electronic currents in the shell create strong magnetic field inside the lightning. These currents are perpendicular to rotational axis, the diameter of rotation decreases to the poles, where magnetic field grows. This retains positive ions from the dispersion along the rotational axis due to the effect of magnetic bottle. Basic magnetic field inside the lightning is directed along the rotational axis. I.e., ions can move along the axis along the lines of magnetic field. From other side, the ions revolve in the circle perpendicularly to axis under the action of Lorentz force with respect to their thermal velocity. As a result at a certain distance from the axis of lightning appears the intersection of two ion flows, which is observed as the luminous shells inside the lightning. Emission from the shells appears from friction and recombination of the being intersected ion flows.

\section{CONCLUSION}

Theory predicts from the first principles the maximum diameter of ball lightning $34 \mathrm{~cm}$. With the larger size the summary charge of lightning, which has positive sign, grows to the value of $10^{-5} \mathrm{C}$ and appears the electrical breakdown of air near the lightning. The energy of the lightning in this case reaches $10.6 \mathrm{~kJ}$, the current in the shell $1.4 \cdot 10^{5} \mathrm{~A}$, the internal magnetic field of 0.5 Tesla.

Because of its charge ball lightning does not simply float under the action of the force of Archimedes, but it is retained by electric force from clouds and the induced charge on the Earth. The formula for the maximum radius of ball lightning has the form:

$$
r=\frac{m c^{2}}{q E_{0}},
$$




\section{REF}

\section{Derivation of the Fermi-Dirac Distribution Function}

We start from a series of possible energies, labeled Ei. At each energy we can have gi possible states and the number of states that are occupied equals gifi, where $f i$ is the probability of occupying a state at energy Ei.

The number of possible ways - called configurations - to fit gi fi electrons in gi states, given the restriction that only one electron can occupy each state, equals:

$$
W_{i}=\frac{g_{i} !}{\left(g_{i}-g_{i} f_{i}\right) ! g_{i} f_{i} !}
$$

This equation is obtained by numbering the individual states and exchanging the states rather than the electrons. This yields a total number of gi! possible configurations. However since the empty states are all identical, we need to divide by the number of permutations between the empty states, as all permutations can not be distinguished and can therefore only be counted once. In addition, all the filled states are indistinguishable from each other, so we need to divide also by all permutations between the filled states, namely gifi!.

The number of possible ways to fit the electrons in the number of available states is called the multiplicity function.

The multiplicity function for the whole system is the product of the multiplicity functions for each energy $E i$

$$
W=\Pi_{i} W_{i}=\prod_{i} \frac{g_{i} !}{\left(g_{i}-g_{i} f_{i}\right) ! g_{i} f_{i} !}
$$

Using Stirling's approximation, one can eliminate the factorial signs, yielding:

$$
\ln W=\sum_{i} \ln W_{i}=\sum_{i}\left[g_{i} \ln g_{i}-g_{i}\left(1-f_{i}\right) \ln \left(g_{i}-g_{i} f_{i}\right)-g_{i} f_{i} \ln g_{i} f_{i}\right]
$$

The total number of electrons in the system equals $N$ and the total energy of those $\mathrm{N}$ electrons equals $\mathrm{E}$. These system parameters are related to the number of states at each energy, gi, and the probability of occupancy of each state, $f i$, by:

$$
\begin{aligned}
& N=\sum_{i} g_{i} f_{i} \\
& \text { and } \\
& \qquad U=\sum_{i} E_{i} g_{i} f_{i}
\end{aligned}
$$

According to the basic assumption of statistical thermodynamics, all possible configurations are equally probable. The multiplicity function provides the number of configurations for a specific set of occupancy probabilities, $f$. The multiplicity function sharply peaks at the thermal equilibrium distribution. The occupancy probability in thermal equilibrium is therefore obtained by finding the maximum of the multiplicity function, $W$, while keeping the total energy and the number of electrons constant.

For convenience, we maximize the logarithm of the multiplicity function instead of the multiplicity function itself. According to the Lagrange method of undetermined multipliers, we must maximize the following function:

$$
\ln W-a \sum_{j} g_{j} f_{j}-b \sum_{j} E_{j} g_{j} f_{j}
$$

where $a$ and $\mathrm{b}$ need to be determined. The maximum multiplicity function is obtained from:

$$
\frac{\partial}{\alpha\left(g_{i} f_{i}\right)}\left[\ln W-a \sum_{j} g_{j} f_{j}-b \sum_{j} E_{j} g_{j} f_{j}\right]=0
$$

American Research Journal of Electronics and Communication Engineering

Page 26 
which can be solved, yielding:

or

$$
\ln \frac{g_{i}-g_{i} f_{i}}{g_{i} f_{i}}-a-b E_{i}=0
$$

$$
f_{i}=f_{F D}\left(E_{i}\right)=\frac{1}{1+\exp \left(a+b E_{i}\right)}
$$

which can be written in the following form

$$
f_{F D}\left(E_{i}\right)=\frac{1}{1+\exp \left(\frac{E_{i}-E_{F}}{, b}\right)}
$$

with $b=1 / b$ and $E F=-a / b$. The symbol $E_{F}$ was chosen since this constant has units of energy and will be the constant associated with this probability distribution.

Taking the derivative of the total energy, one obtains:

Using the Lagrange equation, this can be rewritten as:

$$
d U=\sum_{i} E_{i} d\left(g_{i} f_{i}\right)+\sum_{i} g_{i} f_{i} d E_{i}
$$

$$
d U=, \quad b d(\ln W)+\sum_{i} g_{i} f_{i} d E_{i}+E_{F} d N
$$

Any variation of the energies, Ei, can only be caused by a change in volume, so that the middle term can be linked to a volume variation $d V$.

$$
d U=\beta d(\ln W)+\left[\sum_{i} g_{i} f_{i} \frac{d E_{i}}{d V}\right] d V+E_{F} d N
$$

Comparing this to the thermodynamic identity:

$$
d U=T d S-p d V+\mu d N
$$

The energy, $E_{F}$, equals the energy associated with the particles, $m$.

The comparison also identifies the entropy, $S$, as being the logarithm of the multiplicity function, $W$, multiplied with Boltzmann's constant. The Fermi-Dirac distribution function then becomes:

$$
f_{F D}(E)=\frac{1}{1+\exp \left(\frac{E-E_{F}}{k T}\right)} \text { (C. B. Van Zeghbroeck, } 1998
$$

Citation: Solomon Budnik, "Photonic Displays in Augmented Reality”. American Research Journal of Electronics and Communication Engineering; vol. 1, no. 1; pp: 23-27.

Copyright (C) Solomon Budnik. This is an open access article distributed under the Creative Commons Attribution License, which permits unrestricted use, distribution, and reproduction in any medium, provided the original work is properly cited. 